Article

\title{
Intrabolus Pressure Has Better Correlation Than Eosinophilia with Dysphagia Severity in Fibrostenotic Eosinophilic Esophagitis: A Pilot Study
}

\author{
Jason Colizzo $^{1, *(\mathbb{D}}$, Steven Clayton ${ }^{1}$, Ambuj Kumar ${ }^{2}$ and Joel Richter ${ }^{3}$ \\ 1 Division of Digestive Diseases and Nutrition, Department of Internal Medicine, Morsani College of \\ Medicine, University of South Florida, Tampa, FL 33612, USA; sclayton@ghs.org \\ 2 Department of Evidence Based Medicine and Outcomes Research, Morsani College of Medicine, \\ University of South Florida, Tampa, FL 33612, USA; akumar1@health.usf.edu \\ 3 Joy McCann Culverhouse Center for Swallowing Disorders, Division of Digestive Diseases and Nutrition, \\ Department of Internal Medicine, Morsani College of Medicine, University of South Florida, Tampa, \\ FL 33612, USA; jrichte1@health.usf.edu \\ * Correspondence: jasoncolizzo@me.com; Tel.: +1-813-833-5107
}

Received: 13 July 2018; Accepted: 9 August 2018; Published: 13 August 2018

\begin{abstract}
Eosinophilic esophagitis is characterized by dysphagia with esophageal eosinophilia. We sought to determine if intrabolus pressure measurements on high-resolution manometry had any correlation with dysphagia improvement following standard therapy for patients with fibrostenotic eosinophilic esophagitis. Consecutive patients were prospectively enrolled at our swallowing center. Dysphagia scores, esophageal eosinophil counts, endoscopic reference scores, and intrabolus pressure measurements were compared at baseline and following therapy with 8 weeks of a proton-pump inhibitor and serial bougie dilation to a luminal diameter of $17 \mathrm{~mm}$. Five patients were included in the study. The median age was 38 years. The average endoscopic reference score improved from 5.0 to $2.4(p=0.007)$. The mean esophageal diameter improved from $10.8 \mathrm{~mm}$ to $17.2 \mathrm{~mm}$ $(p=0.001)$. Dysphagia severity scores improved from a mean value of 34.2 to $10.8(p=0.004)$. Mucosal eosinophilia had no correlation with dysphagia severity. Mean intrabolus pressure improved from $21.8 \mathrm{mmHg}$ to $11 \mathrm{mmHg}(p=0.001)$. There was strong correlation between a decrease in intrabolus pressure and improvement in dysphagia severity; however, this was not significant $(p=0.108)$. Intrabolus pressure has strong correlation with dysphagia severity following therapy for fibrostenotic eosinophilic esophagitis. Bougie dilation provides improvement in dysphagia despite persistent mucosal eosinophilia.
\end{abstract}

Keywords: eosinophilic esophagitis; esophageal dilation; high-resolution manometry; dysphagia; endoscopy

\section{Introduction}

Eosinophilic esophagitis (EoE) is a chronic inflammatory condition of the esophagus characterized by the presence of esophageal eosinophilia and dysphagia [1]. The disease initially emerged from case reports in the 1970s and was later recognized more formally as a unique clinical entity in the 1990s [2]. The condition is often diagnosed in young males with Caucasians being the most commonly affected racial group [3]. Incidence rates have been estimated at 2.1-12.8 cases per 100,000 in the USA and appear to be increasing in recent years [4]. The two major characteristics of EoE on endoscopy include inflammatory features (e.g., exudates, edema, furrows) and fibrostenotic features (e.g., rings, strictures, luminal narrowing) [5]. 
The management of EoE is an area of evolving research. Presently, it is common to initially begin patients on an 8-week trial of high-dose proton-pump inhibitor (PPI) therapy to identify PPI responsive EoE [6]. It is estimated that approximately 50\% of patients will respond to this therapy [6,7]. Other therapeutic options include elimination diets, such as the six-food elimination diet (SFED), and topical steroid medications. These three treatments are focused on mitigating ongoing inflammation; however, once fibrosis develops esophageal dilation often proves necessary to relieve the complaint of dysphagia [8-10]. One clinical difficulty is assessing the severity of fibrosis. This feature is often challenging to appreciate with conventional endoscopy, which poorly assesses esophageal narrowing in the range of 10 to $15 \mathrm{~mm}[9,11]$.

Intrabolus pressure (IBP) represents the intraluminal pressure generated to propel a swallowed bolus across the esophagogastric junction (EGJ). There are several factors involved in the generation of the IBP, including bolus size, lumen diameter, force of contraction, and distensibility [12]. In our group's initial retrospective study, we demonstrated that IBP as measured on esophageal high-resolution manometry (HRM) held potential for helping to grade the severity of underlying fibrosis in EoE [13]. Using a cutoff IBP of $16 \mathrm{mmHg}$, we observed a sensitivity of $70.5 \%$ and specificity of $75 \%$ for the presence of fibrostenosis among 12 patients with inflammatory and 17 patients with fibrostenotic EoE. What remained unanswered was whether IBP measurements held any clinical or prognostic utility.

The goal of this pilot study was to prospectively determine the clinical utility of IBP measurements in the management of fibrostenotic EoE. We hypothesized that IBP as measured on repeat HRM would correlate with improvement in dysphagia following standard therapy including a PPI course and dilation. A secondary goal was the assessment of esophageal eosinophilia before and after therapy to determine if improvement in eosinophil burden had any correlation with dysphagia and IBP.

\section{Results}

\subsection{Baseline Demographics and PPI Use}

Five patients were successfully enrolled in the study. Four were males and one patient was female. The mean age of the patients was 38 years (range 27-53 years). Patients had solid food dysphagia for an average of 20.4 years prior to enrollment in our study. Four patients had a history of food impaction. Two patients (40\%) were treated with dexlansoprazole at $60 \mathrm{mg}$ daily, two patients (40\%) were placed on esomeprazole at $40 \mathrm{mg}$ twice daily, and one patient (20\%) on Lansoprazole $30 \mathrm{mg}$ at twice daily (Table 1).

Table 1. Demographics.

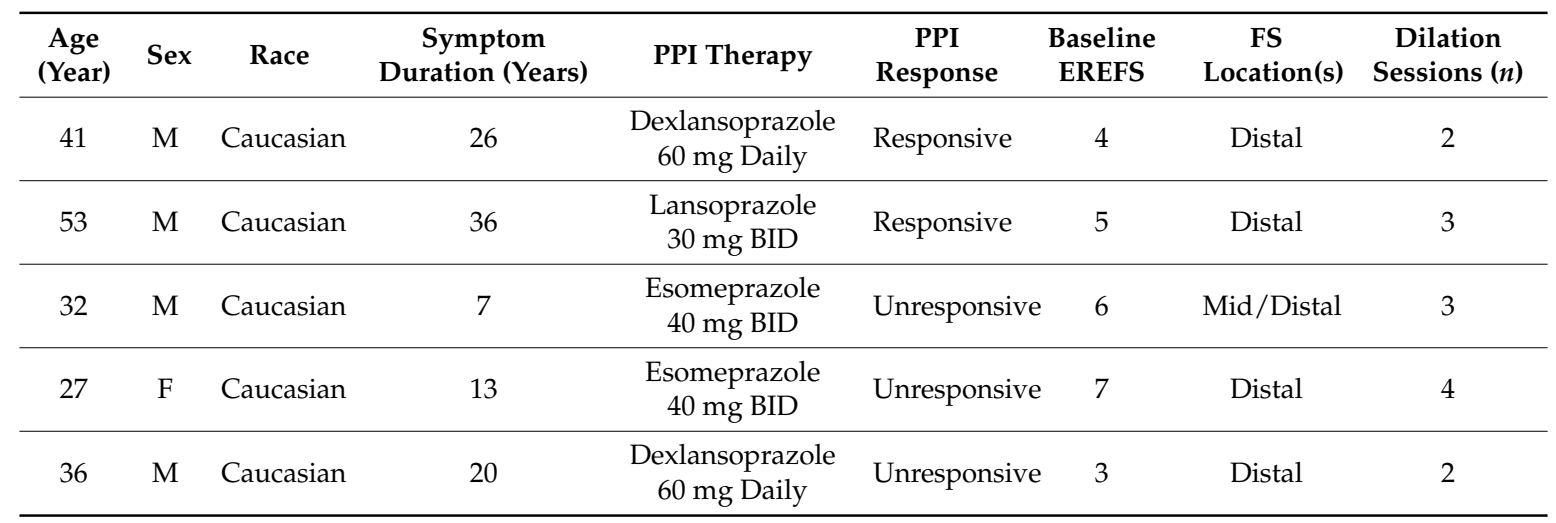

M: male; F: female; PPI: proton-pump inhibitor; BID: twice daily; EREFS: endoscopic reference score; FS: Esophageal fibrostenosis; $n$, number of dilation sessions.

\subsection{Endoscopic Severity and Esophageal Caliber}

At initial endoscopy, the EoE endoscopic reference scoring system (EREFS) was used to calculate a baseline value of disease severity for each patient. The individual EREFS values are demonstrated 
in Table 1. The mean baseline score for EoE severity using this system was 5.0. This improved to a mean value of 2.4 following therapy ( $p=0.007$ ) (Table 2$)$. All patients had baseline esophageal diameters between 8 and $12 \mathrm{~mm}$ (mean $=10.8 \mathrm{~mm}$ ) assessed with Savary or Maloney bougies. Patients underwent serial dilation to $17 \mathrm{~mm}$. The location of esophageal fibrostenosis and the number of dilation sessions required for each patient are illustrated in Table 1. One patient was dilated successfully to an esophageal diameter of $18 \mathrm{~mm}$. The number of dilation sessions ranged from 2 to $4(\mathrm{mean}=2.8)$. The average esophageal diameter improved to 17.2 following dilation therapy $(p=0.001)$ (Table 2). There were no post-procedure complications.

Table 2. Clinical Data Before and After PPIs and Esophageal Dilation.

\begin{tabular}{cccc}
\hline & Baseline $^{*}(\mathrm{SD})$ & Post-Therapy $^{*}$ (SD) & $p$-Value (CI) ${ }^{\dagger}$ \\
\hline Mean eos/hpf proximal esophagus & $57.8(58.8)$ & $26.6(50.2)$ & $0.07(-5.3-67.7)$ \\
Mean eos /hpf distal esophagus & $78.2(58.6)$ & $71.6(107.9)$ & $0.83(-76.3-89.5)$ \\
Esophageal Diameter (mm) & $10.8(1.9)$ & $17.2(2.6)$ & $0.001(-8.4--4.3)^{*}$ \\
USF Dysphagia Score $\ddagger$ & $34.2(12.4)$ & $10.8(6.5)$ & $0.004(12.6-34.2)^{*}$ \\
EREFS Score § & $5(1.5)$ & 2.4 & $0.007(1.1-4.0)^{*}$ \\
IRP mmHg & $8.26(2.7)$ & $6.9(4.2)$ & $0.42(-2.7-5.2)$ \\
DCI mmHg.cm·s & $2319.3(1403.6)$ & $2187.9(1542.5)$ & $0.70(-762.4-1025.1)$ \\
IBP mmHg & $21.8(3.3)$ & $11.0(4.4)$ & $0.001(7.3-14.3)^{*}$ \\
\hline
\end{tabular}

* Values represented as mean; ${ }^{\dagger}$ significance set at $p$-value $0.05 ;{ }^{\ddagger}$ University of South Florida (USF) Dysphagia Score from 0 to 56 in order of increasing severity; $\$$ Endoscopic reference score based on classification by Hirano et al. [5]; SD: standard deviation; CI: 95\% confidence interval; Eos/hpf: eosinophils per high-power field; HRM: high-resolution manometry; IRP: integrated relaxation pressure; DCI: distal contractile integral; IBP: intrabolus pressure.

\subsection{Dysphagia Questionnaire}

All patients had improvement in their solid food dysphagia (Figure 1). The average dysphagia questionnaire score improved from a baseline value of 34.2 to 10.8 following PPIs and dilation therapy, resulting in a mean difference of 23 (95\% CI 12.62 to 34.2). This represents a significant decrease in overall dysphagia score $(p=0.004)$ (Figure 1$)$.

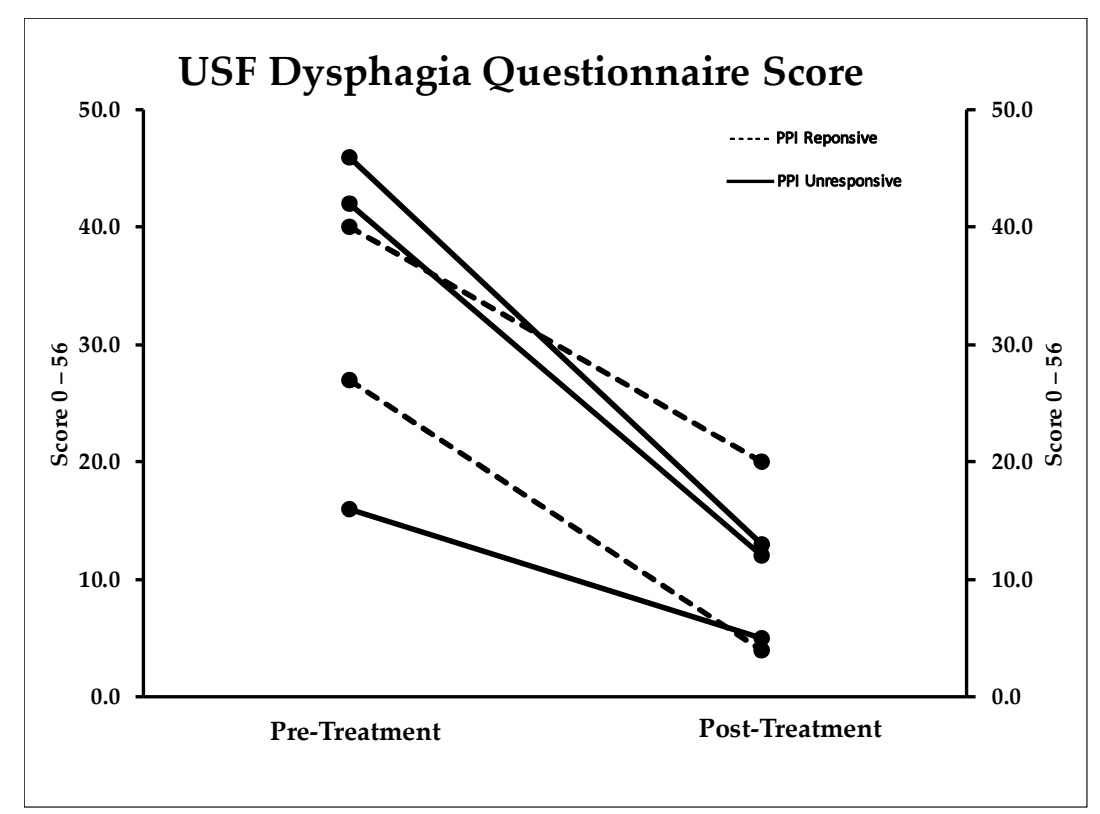

Figure 1. Dysphagia Questionnaire Score. Patients had improvement in their dysphagia scores after esophageal dilation to $16-18 \mathrm{~mm}$ bougie diameter. This was independent of the response to high-dose proton-pump inhibitor therapy for at least 2 months. 


\subsection{Mucosal Eosinophilia}

Biopsies from the distal and proximal esophagus were obtained on all patients at baseline endoscopy and following treatment. The average eosinophil count in the distal esophagus improved from 78.2 to 71.6 after treatment, resulting in a mean difference of 6.6 (95\% CI -76.36 to 89.56). This was not statistically significant $(p=0.836)$. The average eosinophil count in the proximal esophagus improved from 57.8 to 26.6 after treatment, resulting in a mean difference of 31.2 (95\% CI -5.34 to 67.74). This was also not statistically significant $(p=0.077)$. Overall, two of five patients were PPI responders with mucosal eosinophilia decreasing to $<15$ eosinophils/high-power field (hpf) in both biopsies (Table 2). The difference in mucosal eosinophilia in the proximal and distal esophagus following therapy had no significant correlation with improvement in dysphagia scores $(p=0.62$, Correlation coefficient -0.3). Change in mucosal eosinophilia in the proximal esophagus had no correlation with change in IBP ( $p=0.28$, Correlation coefficient -0.6). Change in mucosal eosinophilia in the distal esophagus similarly had no significant correlation with change in IBP $(p=0.50$, Correlation coefficient 0.4 ).

\subsection{Manometry}

All patients had improvement in their IBP, with four falling between normal values $<17 \mathrm{mmHg}$ regardless of change in mucosal eosinophilia. The average baseline IBP improved from 21.85 to 11.00 , resulting in a mean difference of 10.84 (95\% CI 7.36 to 14.32). This was statistically significant $(p=0.001)$ (Figure 2). Other HRM metrics analyzed included integrated relaxation pressure (IRP), bolus clearance, and distal contractile integral (DCI). There was no significant difference between baseline and post-therapy values among these parameters. One patient did exhibit a marked improvement in bolus clearance from $40 \%$ to $100 \%$ and DCI from 1732 to $2216 \mathrm{mmHg} / \mathrm{cm} / \mathrm{s}$ as outlined in the following vignette. There was a strong positive correlation between post-treatment IBP score and Dysphagia scores (Correlation coefficient $=0.8)$. However, this was not statistically significant $(p=0.104)$.

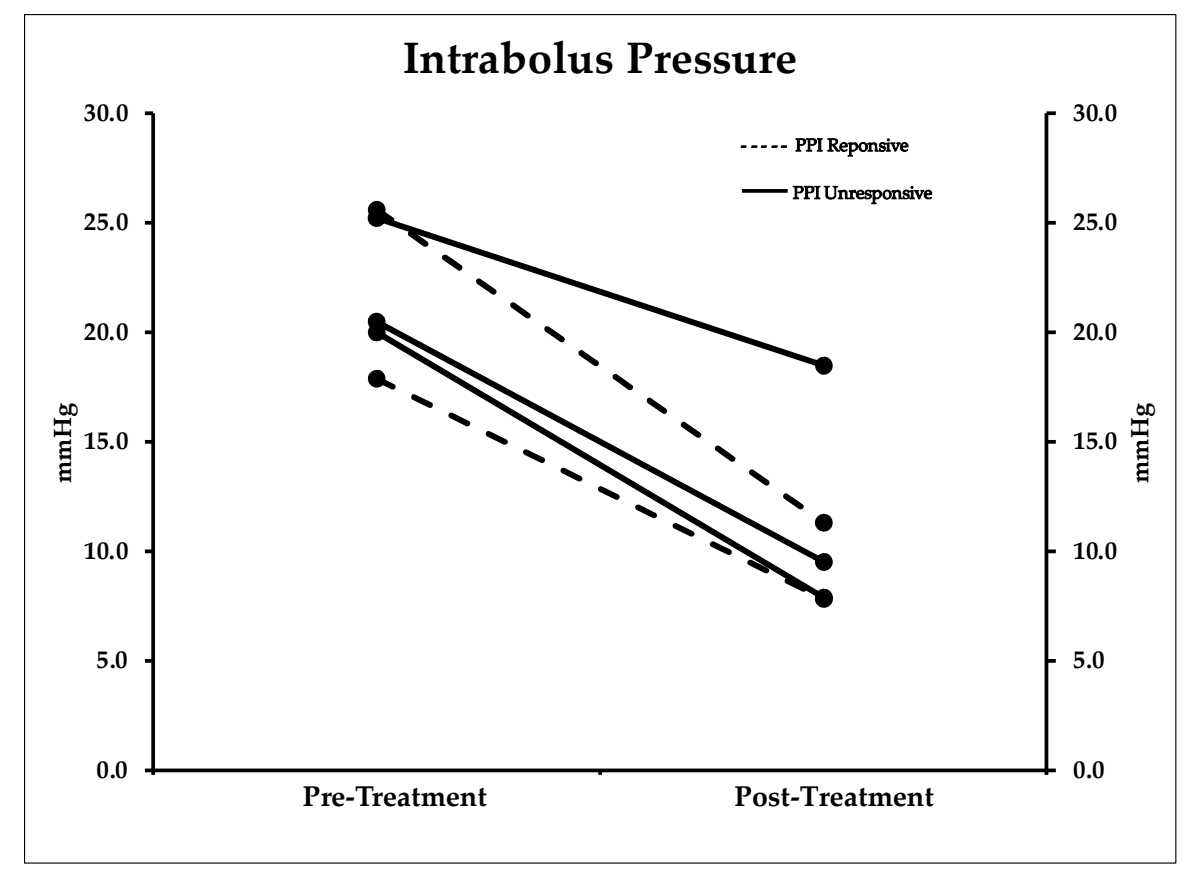

Figure 2. Intrabolus Pressure. Intrabolus pressure improved in all patients after treatment and fell to normal values $(<17 \mathrm{mmHg})$ in four of five patients. Here again this was not dependent on improvement in mucosal eosinophilia. 


\subsection{Clinical Vignette}

A 27-year-old female presented with asthma, selected food allergies (nuts), and solid food dysphagia for a least 13 years. Her initial endoscopy revealed an esophageal luminal diameter of 8-9 $\mathrm{mm}$ assessed via bougie dilation. She also had a baseline EREFS score of 7. Biopsies showed 200 eosinophils/hpf in the distal esophagus and 140 eosinophils/hpf in the proximal esophagus. Baseline HRM found elevated IBP $(25.2 \mathrm{mmHg})$, a DCI of $1732 \mathrm{mmHg} \cdot \mathrm{cm} \cdot \mathrm{s}$, and markedly impaired peristalsis with $60 \%$ failed and $60 \%$ panesophageal pressurization (Figure 3 ).

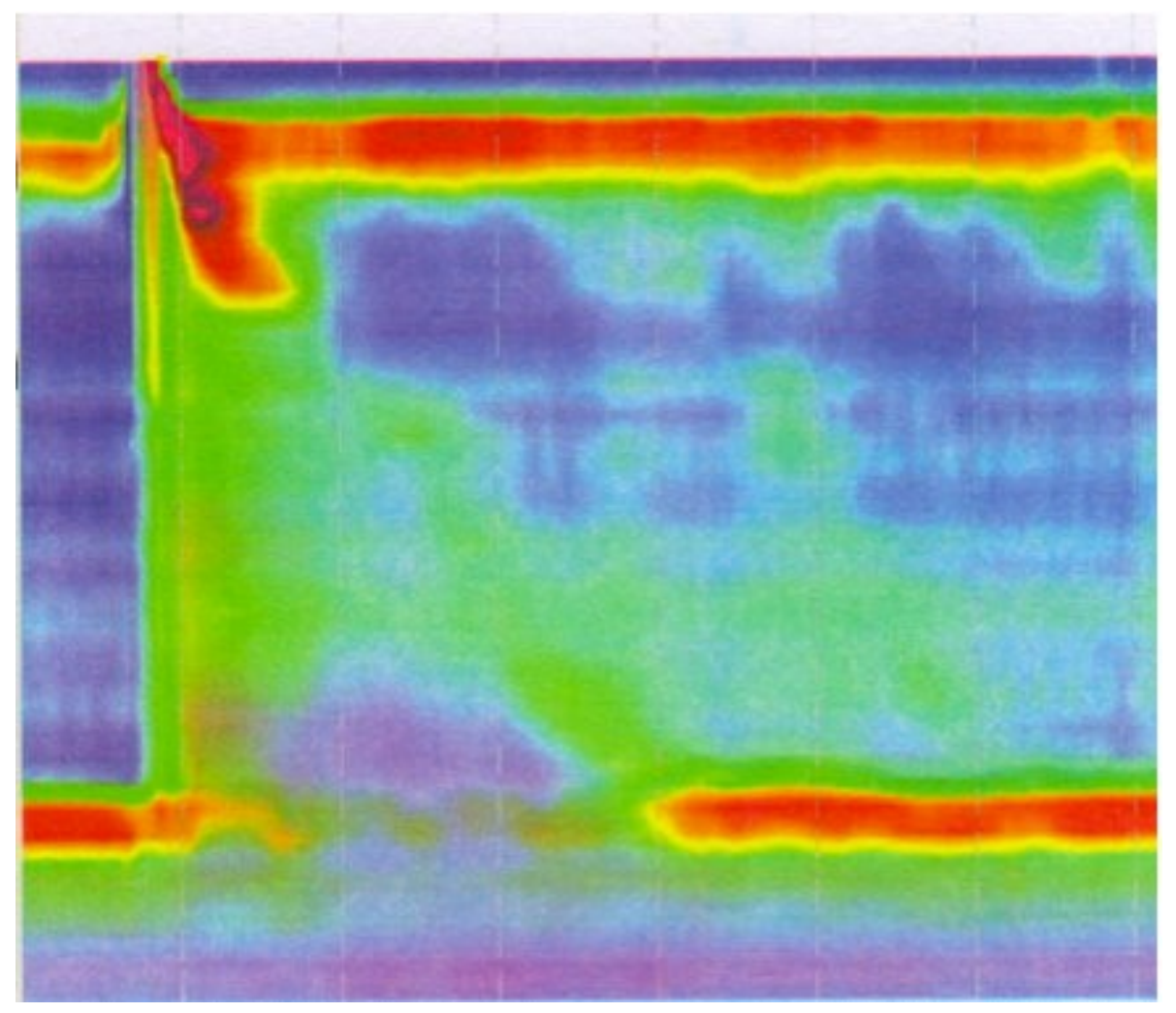

Figure 3. Baseline High-Resolution Esophageal Manometry. Baseline esophageal high-resolution manometry with high intrabolus pressure and impaired peristalsis. IBP 25.2, DCI 1732.9, IRP 5.6, $60 \%$ failed swallows, $60 \%$ Pan-esophageal pressurization.

She was placed on esomeprazole $40 \mathrm{mg}$ twice daily (BID) for 10 weeks and underwent four esophageal dilation sessions increasing her esophageal lumen to $17 \mathrm{~mm}$. Repeat evaluation found marked improvement in her dysphagia score from 46 (severe) to 13 (mild), albeit her mucosal eosinophilia was essentially unchanged (distal 260 and proximal 115 eos/hpf). However, her HRM dramatically improved: IBP decreased to $18.5 \mathrm{mmHg}$, DCI increased to $2216 \mathrm{mmHg} \cdot \mathrm{cm} \cdot \mathrm{s}$, and she demonstrated $100 \%$ normal bolus clearance with no abnormal contractions (Figure 4). Eventually, the patient required topical steroids (budesonide $1 \mathrm{mg}$ BID) to control her mucosal eosinophilia. She has been dysphagia-free for over 3 years and has required only one subsequent esophageal dilation. 


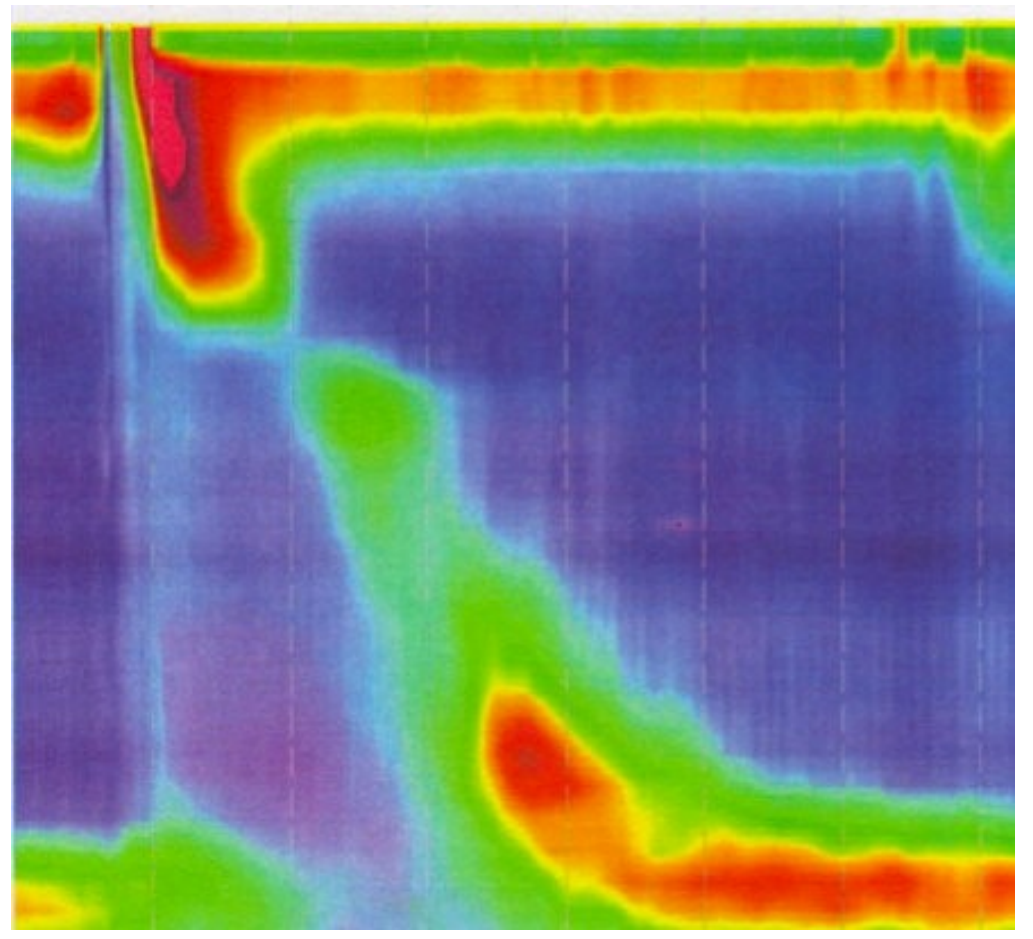

Figure 4. High-Resolution Esophageal Manometry Following Therapy. High-resolution esophageal manometry following therapy including 3 months of twice-daily PPI and esophageal dilation. This demonstrates normalization of IBP and peristalsis. Esophageal diameter increased nearly $100 \%$ to $17 \mathrm{~mm}$. This improvement suggests that fibrotic scarring was trapping the esophageal body, preventing normal peristalsis and bolus clearance.

\section{Discussion}

Our findings from this pilot study suggest that IBP could be a potential marker of disease severity in patients with fibrostenotic EoE. Patients in our study were treated with the customary 8-week course of PPI therapy. This yielded improvement in mucosal eosinophilia in only two of the five patients (40\%). Despite the fact that most of our patients had persistent eosinophil burden following PPI therapy, all patients had a significant improvement in dysphagia severity following esophageal dilation. This clinical improvement showed a strong correlation (correlation coefficient $=0.8$ ) with a reduction in IBP to normal values.

The IBP is one of several parameters analyzed by HRM. While the contractile wave helps to identify major motility disturbances, it may not account for other changes occurring within the esophageal wall that may be contributing to dysphagia. As shown by Nguyen et al. in 42 patients with non-obstructive dysphagia, IBP and IBP slope were significantly higher in non-obstructive dysphagia patients than in healthy controls regardless of the presence of non-specific motility patterns [14]. In another study by Kou et al., the importance of IBP was highlighted in a computational model of esophageal transport [15]. In this study, mucosal stiffness was associated with a rise in IBP and a decrease in bolus transit efficiency. Their findings emphasize the importance esophageal remodeling can have in deglutition, which is also illustrated by our case vignette. Such esophageal changes may not be fully illustrated with other HRM parameters. In our study population, the presence of esophageal remodeling in the form of fibrotic changes due to longstanding EoE is the driving factor in the high IBP pressures we recorded.

It has been proposed that the fibrostenotic form of EoE is secondary to long-standing inflammation. For our patient population, the average symptom duration prior to diagnosis was 20.4 years. Schoepfer et al. first demonstrated this point in a 2013 study in which the delay in diagnosis of 
EoE was associated with fibrotic features including strictures [16]. The experience of our Swallowing Center at the University of South Florida has been similar. We observed a significant association between decreasing esophageal diameter and diagnostic delay with nearly $50 \%$ of EoE patients having an esophageal stricture by 10 years and $80 \%$ by 20 years of untreated disease [17]. These observations suggest that treatment, when initiated early in the disease course, may delay the progression of fibrosis and esophageal narrowing.

Traditional treatments for EoE concentrate on interventions to decrease the eosinophil burden within the esophagus. It remains to be determined which therapy (PPI, topical steroids, SFED) has the greatest potential for decreasing inflammation and preventing fibrostenotic changes over time. Long-term prospective data on the natural history of EoE and management strategies are currently needed. Our small pilot study illustrates that inflammation alone may be less of a factor in EoE-related dysphagia than esophageal fibrostenosis. This is supported by our observation in three of our patients whose mucosal eosinophilia remained unchanged while their dysphagia markedly improved following dilatation. Other studies, such as Alexander et al., found a similar discordance between esophageal eosinophilia and dysphagia severity [18]. These results question the significance of histologic response alone in EoE, particularly in those patients with fibrostentotic disease. In the presence of fibrostenosis, esophageal dilatation is required to provide long-lasting improvement of dysphagia despite ongoing mucosal eosinophilia [19-21].

One of the primary changes underlying the response to dilation therapy is the disruption in the fibrotic scar followed by remodeling. Studies incorporating full-thickness biopsies have found increased fibrosis in the lamina propria [22]. TGF- $\beta$, a cytokine shown to induce fibroblast activation, appears to play a role in the development of esophageal fibrogenesis [23]. A study conducted by Persad et al. similarly concluded that esophageal biopsies from EoE patients had higher levels of TGF- $\alpha$ over healthy controls [24]. Other pro-angiogeneic factors, such as VEGF-A, angiogenin, and IL-8, were also found in the EoE group [24]. These and other studies help to highlight the fact that significant changes beneath the mucosal surface may lead to clinical consequences with time.

While endoscopy can identify obvious fibrotic features, measuring the true degree of esophageal lumen narrowing in EoE remains a challenge. Studies have determined the barium esophagram often detects symptomatic esophageal narrowing not recognized on endoscopy. The Mayo group observed that endoscopy, when compared to a detailed barium esophagram, had poor sensitivity $(25 \%)$ in defining strictures in the symptomatic range from 10 to $15 \mathrm{~mm}$ diameter [11]. We agree and therefore routinely carefully dilate all patients with EoE to assess the true lumen diameter defined by the bougie size at which resistance is noted with passage of Savary or Maloney dilators. Another proposed tool to help in assessing the degree of fibrosis is the functional lumen imaging probe (FLIP). This instrument obtains esophageal metrics simultaneously to assess esophageal distensibility [25]. In a study by Nicodeme et al., reduced esophageal distensibility as detected by FLIP technology was associated with an increased risk for food impactions in EoE patients [26]. Future studies incorporating FLIP technology and HRM may help us better understand the role of esophageal diameter as it relates to IBP and esophageal distensibility.

Our pilot study is limited primarily by our small sample size. This was due in large part to the fact that patients were reluctant to repeat manometry after their dysphagia resolved. Another limiting factor included the recent Chicago Classification (3.0), which does not contain IBP measurements. It is disappointing that IBP is no longer routinely available for HRM studies as it appears to be the manometric feature in our study that correlated best with the degree of esophageal fibrosis and clinical improvement following esophageal dilation. Nevertheless, this pilot study reconfirms that mucosal eosinophilia may be a suboptimal treatment endpoint in the setting of fibrostenosis.

\section{Materials and Methods}

We performed a prospective cohort pilot study where all consecutive patients aged 18 to 90 were prospectively enrolled at the Joy McCann Culverhouse Center for Swallowing Disorders if they 
had biopsy-confirmed EoE with at least 15 eosinophils per high-power field (HPF) in the proximal and distal esophagus, endoscopic evidence of fibrostenosis defined as esophageal diameter $<17 \mathrm{~mm}$ on endoscopy and ease of passing bougies throughout the esophagus, and complaints of solid food dysphagia. The severity of EoE on endoscopy was assessed using the EoE endoscopic reference scoring system (EREFS) developed by Hirano et al. [5]. In this classification system, the severity of EoE is graded using features such as rings, exudates, furrows, edema, strictures, and crepe paper esophagus on a scale from 0 to 9 in order of increasing severity [5]. Informed consent was obtained from all study participants. Our study (Pro00016178; 14 October 2014) was approved by the Institutional Review Board at the University of South Florida (USF).

All patients underwent baseline HRM utilizing esophageal manometry catheters and acquisition software from Medtronics, INC ${ }^{\mathrm{TM}}$ in Minneapolis, MN. Patients were enrolled only if the IBP was at least $17 \mathrm{mmHg}$ based on the Chicago 2.0 analysis program. This value was chosen from our previous retrospective study by which an IBP $>16 \mathrm{mmHg}$ indicated fibrostenotic features on endoscopy with a sensitivity and specificity of $70.5 \%$ and $75 \%$, respectively [13].

All patients completed a validated dysphagia questionnaire at our Swallowing Center using a modification of the EoE scoring system developed by Schoepfer et al. with their permission (Appendix A) [27]. The University of South Florida (USF) dysphagia questionnaire includes two questions assessing quality of life over the last 14 days that are scored from 0 (no dysphagia) to 4 (severe dysphagia). A third unscored question was used to screen for other disorders that may mask symptoms, such as oropharyngeal pain and recent dental infections. If such disorders were present, the patient was excluded from the study. A visual food scale was then provided consisting of eight foods of various textures (steak, pudding, ground beef, rice, apple, bread, oatmeal, French fries).

Patients were asked to grade the severity and frequency of dysphagia for each food (Table A1). Dysphagia severity was scored from 0 (no difficulty) to 3 (severe difficulty). The frequency of dysphagia for each food was also scored from 0 (never) to 3 (always). The scores from the first two quality of life questions and the severity and frequency of dysphagia for each food item were summed to create the final composite score of 0 (no dysphagia) to 56 (severe dysphagia).

All patients were placed on twice-daily PPI therapy. The specific PPI used was determined according to each patient's insurance coverage and included esomeprazole, lansoprazole, or dexlansoprazole. They underwent esophagogastroduodenoscoy (EGD) with graduated bougienage dilation every 3-4 weeks after enrollment until an esophageal diameter of 16-17 mm was achieved. After 8-12 weeks, HRM and EGD with biopsies were repeated to reassess IBP values and eosinophil burden, respectively. A second dysphagia questionnaire was also given.

\section{Statistical Analysis}

Patient characteristics and demographic variables were summarized either as mean and standard deviations for continuous variables and as rates for categorical variables. The changes in continuous variables pre and post treatment were assessed using a paired samples $t$-test. The correlation between variables was assessed using the non-parametric Spearman correlation test. The significance level for all comparisons was set at $5 \%$. All analyses were performed using SPSS statistical analysis software (Version 24, IBM Co., Armonk, NY, USA).

\section{Conclusions}

In our small pilot study of adult patients with fibrostenotic EoE, our findings reveal that bougie dilation can lead to excellent improvement in dysphagia severity despite persistent mucosal eosinophilia. IBP demonstrated a stronger correlation with improvement in dysphagia than mucosal eosinophilia. Therapies including PPIs and topical steroids can help mitigate ongoing inflammation and may help to decrease stricture reformation.

Author Contributions: Conceptualization, J.C., S.C. and J.R.; Investigation, J.C., S.C. and J.R.; Data curation, J.C. and A.K.; Formal analysis, A.K.; Writing (Original Draft Preparation), J.C. and J.R.; Supervision, J.R. 
Funding: This research received no external funding.

Acknowledgments: There are no funding sources to disclose. The authors confirm there are no conflicts of interest. The study team would like to thank Betsy Lamoy, Dawn Teague, and Nicole Wood for their assistance with patient care during this study.

Conflicts of Interest: The authors declare no conflict of interest

\section{Appendix A. University of South Florida (USF) Dysphagia Questionnaire}

Please check an answer box for questions 1-3 regarding your health and diet over the last 14 days:

(1) Have you experienced any of the following symptoms?

$\begin{array}{lll}\text { Sore throat } & \text { Cough } & \text { Tooth/oral pain while eating } \\ \text { Fever } & \text { Nasal congestion } & \end{array}$

Yes (if yes, please circle answer above)

No

(2) How often do you currently experience difficulty swallowing food?

Very frequently (nearly every meal)

Frequently (3-5 days/week)

Sometimes (1-2 days/week)

Rarely (1-2 meals/14-days)

Never

(3) Which answer choice best describes the impact eosinophilic esophagitis (EoE) has had on your life?

I worry about choking with every meal

Trouble swallowing has caused me to avoid certain foods

I am able to eat most foods, but have to modify their consistency

I am able to eat most foods, and rarely experience trouble swallowing

I am able to eat food without any difficulty swallowing or change in diet

(4) On the following page, please carefully examine each of the following food items listed and:

(A) Indicate how difficult it would be to swallow each of the following food items without changing its consistency (e.g., using food processor, crushing, liquefying, etc.)

No difficulty: I Can eat this food without any trouble swallowing

Minimal difficulty: I experience some difficulty when swallowing this food

Moderate difficulty: It takes some time to completely swallow this food

Severe difficulty: I experience extreme difficulty when swallowing this food. I worry about choking with this food.

(B) Indicate how frequently you would experience difficulty in swallowing each of the food items without changing its consistency (e.g., using food processor, crushing, liquefying, etc.)

Never have any problems: 0 episodes of swallowing difficulties with this food

Rarely: $<2$ episodes/week of swallowing difficulty with this food

Frequently: 3-5 episodes/week of swallowing difficulty with this food

Always: Experience difficulty swallowing this food every time 
Table A1. University of South Florida Dysphagia Questionnaire.

\begin{tabular}{cllllll}
\hline & & & &
\end{tabular}




\section{References}

1. Patel, R.V.; Hirano, I. New developments in the diagnosis, therapy, and monitoring of eosinophilic esophagitis. Curr. Treat. Options Gastroenterol. 2018, 16, 15-26. [CrossRef] [PubMed]

2. Straumann, A.; Spichtin, H.P.; Bernoulli, R.; Loosli, J.; Vogtlin, J. [idiopathic eosinophilic esophagitis: A frequently overlooked disease with typical clinical aspects and discrete endoscopic findings]. Schweiz. Med. Wochenschr. 1994, 124, 1419-1429. [PubMed]

3. Mansoor, E.; Cooper, G.S. The 2010-2015 prevalence of eosinophilic esophagitis in the USA: A population-based study. Dig. Dis. Sci. 2016, 61, 2928-2934. [CrossRef] [PubMed]

4. Dellon, E.S.; Hirano, I. Epidemiology and natural history of eosinophilic esophagitis. Gastroenterology 2017, 154, 319-332. [CrossRef] [PubMed]

5. Hirano, I.; Moy, N.; Heckman, M.G.; Thomas, C.S.; Gonsalves, N.; Achem, S.R. Endoscopic assessment of the oesophageal features of eosinophilic oesophagitis: Validation of a novel classification and grading system. Gut 2013, 62, 489-495. [CrossRef] [PubMed]

6. Molina-Infante, J.; Prados-Manzano, R.; Gonzalez-Cordero, P.L. The role of proton pump inhibitor therapy in the management of eosinophilic esophagitis. Expert Rev. Clin. Immunol. 2016, 12, 945-952. [CrossRef] [PubMed]

7. Moawad, F.J.; Cheng, E.; Schoepfer, A.; Al-Haddad, S.; Bellizzi, A.M.; Dawson, H.; El-Zimaity, H.; Guindi, M.; Penagini, R.; Safrooneva, E.; et al. Eosinophilic esophagitis: Current perspectives from diagnosis to management. Ann. N. Y. Acad. Sci. 2016, 1380, 204-217. [CrossRef] [PubMed]

8. Lipowska, A.M.; Kavitt, R.T. Current diagnostic and treatment strategies for eosinophilic esophagitis. Gastroenterol. Hepatol. 2017, 13, 527-535.

9. Richter, J.E. Endoscopic treatment of eosinophilic esophagitis. Gastrointest. Endosc. Clin. N. Am. 2018, 28, 97-110. [CrossRef] [PubMed]

10. Moawad, F.J.; Molina-Infante, J.; Lucendo, A.J.; Cantrell, S.E.; Tmanova, L.; Douglas, K.M. Systematic review with meta-analysis: Endoscopic dilation is highly effective and safe in children and adults with eosinophilic oesophagitis. Aliment. Pharmacol. Ther. 2017, 46, 96-105. [CrossRef] [PubMed]

11. Gentile, N.; Katzka, D.; Ravi, K.; Trenkner, S.; Enders, F.; Killian, J.; Kryzer, L.; Talley, N.J.; Alexander, J. Oesophageal narrowing is common and frequently under-appreciated at endoscopy in patients with oesophageal eosinophilia. Aliment. Pharmacol. Ther. 2014, 40, 1333-1340. [CrossRef] [PubMed]

12. Quader, F.; Reddy, C.; Patel, A.; Gyawali, C.P. Elevated intrabolus pressure identifies obstructive processes when integrated relaxation pressure is normal on esophageal high-resolution manometry. Am. J. Phys. Gastrointest. Liver Phys. 2017, 313, G73-G79. [CrossRef] [PubMed]

13. Colizzo, J.M.; Clayton, S.B.; Richter, J.E. Intrabolus pressure on high-resolution manometry distinguishes fibrostenotic and inflammatory phenotypes of eosinophilic esophagitis. Dis. Esophagus 2016, 29, 551-557. [CrossRef] [PubMed]

14. Nguyen, N.Q.; Holloway, R.H.; Smout, A.J.; Omari, T.I. Automated impedance-manometry analysis detects esophageal motor dysfunction in patients who have non-obstructive dysphagia with normal manometry. Neurogastroenterol. Motil. 2013, 25, 238-245. [CrossRef] [PubMed]

15. Kou, W.; Pandolfino, J.E.; Kahrilas, P.J.; Patankar, N.A. Simulation studies of the role of esophageal mucosa in bolus transport. Biomech. Model. Mechanobiol. 2017, 16, 1001-1009. [CrossRef] [PubMed]

16. Schoepfer, A.M.; Safroneeva, E.; Bussmann, C.; Kuchen, T.; Portmann, S.; Simon, H.U.; Straumann, A. Delay in diagnosis of eosinophilic esophagitis increases risk for stricture formation in a time-dependent manner. Gastroenterology 2013, 145, 1230-1236. [CrossRef] [PubMed]

17. Lipka, S.; Kumar, A.; Richter, J.E. Impact of diagnostic delay and other risk factors on eosinophilic esophagitis phenotype and esophageal diameter. J. Clin. Gastroenterol. 2016, 50, 134-140. [CrossRef] [PubMed]

18. Alexander, J.A.; Jung, K.W.; Arora, A.S.; Enders, F.; Katzka, D.A.; Kephardt, G.M.; Kita, H.; Kryzer, L.A.; Romero, Y.; Smyrk, T.C.; et al. Swallowed fluticasone improves histologic but not symptomatic response of adults with eosinophilic esophagitis. Clin. Gastroenterol. Hepatol. 2012, 10, 742-749. [CrossRef] [PubMed]

19. Bohm, M.; Richter, J.E.; Kelsen, S.; Thomas, R. Esophageal dilation: Simple and effective treatment for adults with eosinophilic esophagitis and esophageal rings and narrowing. Dis. Esophagus 2010, 23, 377-385. [CrossRef] [PubMed] 
20. Richter, J.E. Esophageal dilation in eosinophilic esophagitis. Best Pract. Res. Clin. Gastroenterol. 2015, 29, 815-828. [CrossRef] [PubMed]

21. Moole, H.; Jacob, K.; Duvvuri, A.; Moole, V.; Dharmapuri, S.; Boddireddy, R.; Uppu, A.; Puli, S.R. Role of endoscopic esophageal dilation in managing eosinophilic esophagitis: A systematic review and meta-analysis. Medicine 2017, 96, e5877. [CrossRef] [PubMed]

22. Mueller, S.; Aigner, T.; Neureiter, D.; Stolte, M. Eosinophil infiltration and degranulation in oesophageal mucosa from adult patients with eosinophilic oesophagitis: A retrospective and comparative study on pathological biopsy. J. Clin. Pathol. 2006, 59, 1175-1180. [CrossRef] [PubMed]

23. Nguyen, N.; Furuta, G.T.; Masterson, J.C. Deeper than the epithelium: Role of matrix and fibroblasts in pediatric and adult eosinophilic esophagitis. J. Pediatr. Gastroenterol. Nutr. 2016, 63, 168-169. [CrossRef] [PubMed]

24. Persad, R.; Huynh, H.Q.; Hao, L.; Ha, J.R.; Sergi, C.; Srivastava, R.; Persad, S. Angiogenic remodeling in pediatric EoE is associated with increased levels of VEGF-A, angiogenin, IL-8, and activation of the TNF-alpha-NFKappaB pathway. J. Pediatr. Gastroenterol. Nutr. 2012, 55, 251-260. [CrossRef] [PubMed]

25. Hirano, I.; Pandolfino, J.E.; Boeckxstaens, G.E. Functional lumen imaging probe for the management of esophageal disorders: Expert review from the clinical practice updates committee of the aga institute. Clin. Gastroenterol. Hepatol. 2017, 15, 325-334. [CrossRef] [PubMed]

26. Nicodeme, F.; Hirano, I.; Chen, J.; Robinson, K.; Lin, Z.; Xiao, Y.; Gonsalves, N.; Kwasny, M.J.; Kahrilas, P.J.; Pandolfino, J.E. Esophageal distensibility as a measure of disease severity in patients with eosinophilic esophagitis. Clin. Gastroenterol. Hepatol. 2013, 11, 1101-1107. [CrossRef] [PubMed]

27. Schoepfer, A.M.; Straumann, A.; Panczak, R.; Coslovsky, M.; Kuehni, C.E.; Maurer, E.; Haas, N.A.; Romero, Y.; Hirano, I.; Alexander, J.A.; et al. Development and validation of a symptom-based activity index for adults with eosinophilic esophagitis. Gastroenterology 2014, 147, 1255-1266. [CrossRef] [PubMed]

(C) 2018 by the authors. Licensee MDPI, Basel, Switzerland. This article is an open access article distributed under the terms and conditions of the Creative Commons Attribution (CC BY) license (http:/ / creativecommons.org/licenses/by/4.0/). 